\title{
Energy Dissipation in Nonlinear Systems Coupled to a Bath: On the Use of Perturbative Maps
}

\author{
Eran Rabani and B. J. Berne* \\ Department of Chemistry and Center for Biomolecular Simulation, Columbia University, 3000 Broadway, \\ New York, New York 10027
}

Received: March 11, 1998; In Final Form: May 22, 1998

\begin{abstract}
Iterated maps, which mimic the motion of nonlinear systems coupled to a bath, are described in the weak coupling regime. Classical time-dependent perturbation theory is used to derive the maps. To study vibrational energy transfer, the system is modeled by the harmonic and Morse oscillators. For chemical reactions the system is described by the double-well potential. Particular attention is given to the coupling between the systems and the bath, which is taken to be nonlinear in the bath modes. The maps provide a very efficient way to numerically simulate the dynamics of the systems, but are unique in their ability to delineate the various coupling parameters that govern the dynamics. A simple "random phase" limit of the maps is discussed and leads to a kinetic description of the dynamics given by a multidimensional Fokker-Planck equation. Explicit expressions for the energy-diffusion coefficients are obtained.
\end{abstract}

\section{Introduction}

The pioneering work of Kramers ${ }^{1}$ treats the process of crossing a barrier as one that is governed by Brownian dynamics, in which the surrounding molecules play a major role. It is a well-recognized fact that the process of crossing a barrier in condensed phases is a rare event which requires special theoretical treatment. ${ }^{2-19}$ Many of the theoretical approaches have been comprehensively reviewed by Hänggi, Talkner, and Borkovec. $^{20}$ The coupling between the solvent and solute degrees of freedom means that even when the products are well separated and outside the region of interaction, they are not isolated. Many chemical reactions in condensed phases are accompanied by relaxation processes, and these have received considerable attention by several theoretical groups. ${ }^{21-38}$

Chemical reactions in condensed phases are frequently described using two different methods. The simple approach consists of a Brownian particle moving in a one-dimensional bistable potential; the dynamics of this system are described by the Langevin equation. ${ }^{1}$ Grote and Hynes ${ }^{9}$ have extended this approach to non-Markovian processes for a parabolic barrier, where the dynamics are given by the generalized Langevin equation. ${ }^{39}$ At almost the same time, Carmeli and Nitzan ${ }^{40}$ generalized Kramers' model to include memory effects in the weak damping regime. These and other theories were tested numerically by Straub, Borkovec, and Berne, 6,7 and their results were crucial in the development of the Pollak, Grabert, Hänggi turnover theory. ${ }^{14}$ All of these theories assume a Gaussian random force for the generalized Langevin equation. In this limit, the dynamics can be transformed into a Hamiltonian description where the system is linearly coupled to a harmonic bath. ${ }^{41}$

The other approach is based on molecular dynamics simulations, which can account for a more general form of the Hamiltonian. Special methods have been developed to accelerate the barrier crossing and thereby numerically determine the rate constant. Chandler ${ }^{2}$ showed that in the time-correlation approach to rate constants ${ }^{42,43}$ the reactive flux rapidly decays to a plateau value, which can then be associated with the slow rate for crossing the barrier. ${ }^{3,5}$ Straub, Hsu, and Berne have introduced an absorbing boundaries method for calculating the reactive flux in the energy diffusion regime. ${ }^{44,45}$ Charutz and Levine $^{46-48}$ have made a successful attempt to separate the relaxation processes from the actual barrier crossing in condensed phases and thus clarify and simplify our understanding of the role of the solvent molecules in chemical reaction. Their approach is based on the use of "dressed" variables, ${ }^{47}$ which share many common features of the ordinary gas-phase variables. With the help of these dressed variables, it is possible to compute those quantities that characterize the dynamics in the gas phase, ${ }^{49}$ such as the product energy distribution and state to state rates.

In this paper we link the simple stochastic approach with molecular dynamics for systems in the weak coupling regime. Motivated by the work of Zwanzig, ${ }^{21}$ we develop a new theoretical method based on the reduction of the Hamiltonian dynamics to an iterated map. ${ }^{50-53}$ This treatment has been extensively applied to the theoretical study of the excitation and ionization of hydrogen atoms by microwaves, ${ }^{54-56}$ and recently, Rabani and Levine have applied it to the dynamics of high molecular Rydberg states. ${ }^{57,58}$

We consider a rather simple, yet realistic, model Hamiltonian for a system embedded in a condensed-phase environment, which is described in section II. Three different systems are studied; for vibrational energy transfer the system is described by the linear harmonic or the nonlinear Morse oscillators, and for chemical reaction the system is described by the doublewell model. The bath is modeled by an effective harmonic Hamiltonian (a collection of harmonic modes) ${ }^{37}$ which can be fit to a more realistic model, such as a solvent whose molecules interact through the pairwise Lennard-Jones potential. ${ }^{59}$ The coupling between the system and the bath modes is taken to be nonlinear in the bath modes (i.e., it is expanded in a Taylor series), so that our model can account for multiphonon energy transfer and is more general than the stochastic approach. Egorov and Berne obtained a quantum mechanical solution using first-order perturbation theory for a similar model, where the 
primary system was a linear harmonic oscillator. ${ }^{59}$ They found this model to be general enough to capture the essential features of vibrational energy relaxation.

The reduction of the Hamiltonian description of the motion into a map is discussed in section III. This reduction is based on first-order perturbation theory, ${ }^{50-53}$ which is an adequate approximation to the dynamics in the weak coupling regime. The other ingredient needed for this reduction is the transformation of the conventional set of variables to action-angle variables. ${ }^{50,60}$ This transformation is required by classical perturbation theory and is the limiting step in the current approach. Nevertheless, we show how approximate actionangle variables ${ }^{50}$ do the job equally well. The resultant map specifies the changes in the values of the system-bath actionangle variables after several periods of the system. We note in passing that Cortés, West, and Lindenberg ${ }^{29}$ have applied classical perturbation theory to a large class of system-bath interaction potentials. However, their implementation of the perturbation theory is very different from the present approach. ${ }^{29}$

In section IV, we invoke a "random phase" approximation, which provides a way to further simplify the map. In this limit, the description of the motion given by the map can be immediately reduced to a Fokker-Planck equation in action space. ${ }^{61,62}$ We discuss the validity of this approximation and derive analytical expressions for the action-dependent diffusion coefficients. $^{40}$

In section $\mathrm{V}$, we assess the accuracy of the map. We show that the dynamics generated by numerically iterating the map are in excellent agreement with the Hamiltonian dynamics for all three systems. Moreover, it is shown that the kinetic description given by the Fokker-Planck equation provides a realistic approximation to the numerical results. Concluding remarks are given in section VI.

\section{Model Hamiltonian}

We consider a simple model Hamiltonian that describes the interactions of a system with a bath. Only systems with one degree of freedom are studied here. Multidimensional systems are left for future study. For vibrational energy transfer, the system is described by either a harmonic or a Morse oscillator. The double-well potential is used to model a chemical reaction. The bath is modeled by an ensemble of harmonic oscillators, which amounts to an effective harmonic Hamiltonian. ${ }^{37}$ The coupling between the system and the bath is taken to be linear in the system mode and is expanded in a Taylor series in the bath modes.

Throughout this paper we use lower case symbols for the system variables and upper case symbols for the bath variables. The total Hamiltonian can be written as

$$
H=h(q)+H_{\mathrm{b}}(\mathbf{Q})+V(q, \mathbf{Q})
$$

where $h(q)$ is the Hamiltonian for the system,

$$
h(q)=\frac{1}{2} p^{2}+V(q)
$$

In eq 2, $q$ is the mass-weighted system coordinate with conjugate momentum $p$, and $V(q)$ is the potential energy. As mentioned before, we consider the following three forms for $V(q)$; for the harmonic oscillator we have

$$
V(q)=\frac{1}{2} \omega^{2} q^{2}
$$

where $\omega$ is the vibrational frequency. For the nonlinear Morse oscillator the potential is given by

$$
V(q)=D\left(1-\mathrm{e}^{-\beta \mathrm{q}}\right)^{2}
$$

where $D$ and $\beta$ are the well depth and the mass-weighted range parameters of the Morse function, respectively. The doublewell potential is

$$
V(q)=\frac{1}{2} k q^{2}+c q^{3}+d q^{4}
$$

where $c$ and $d$ are the expansion coefficients. For reasons that will become clear in the Appendix, $k$ is taken to be positive and not negative, as is commonly the case for the standard symmetric representation of the double-well potential.

The bath Hamiltonian in the harmonic approximation takes the form

$$
H_{\mathrm{b}}(\mathbf{Q})=\frac{1}{2} \sum_{\alpha}^{N_{\mathrm{b}}} P_{\alpha}{ }^{2}+\frac{1}{2} \sum_{\alpha}^{N_{\mathrm{b}}} \omega_{\alpha}{ }^{2} Q_{\alpha}{ }^{2}
$$

where the summation index $\alpha$ labels the mass-weighted bath coordinates $Q_{\alpha}$, which have conjugate momenta $P_{\alpha}$, and frequencies $\omega_{\alpha} ; N_{\mathrm{b}}$ is the number of bath modes.

As mentioned previously, the system-bath coupling is taken to be linear in the system coordinate and is expanded in a Taylor series in the bath coordinates. ${ }^{59}$ For clarity and simplicity we neglect all off-diagonal terms in the Taylor expansion. (The generalization of the present treatment to account for such terms is rather straightforward, but is left for future study.) In the present work the coupling is given by

$$
V(q, \mathbf{Q})=q f(\mathbf{Q})
$$

where $f(\boldsymbol{Q})$ is the Taylor expansion of the coupling in the bath coordinates (neglecting the off-diagonal terms),

$$
f(\mathbf{Q})=\sum_{\alpha}^{N_{\mathrm{b}}} g_{\alpha} Q_{\alpha}+\sum_{\alpha}^{N_{\mathrm{b}}} h_{\alpha} Q_{\alpha}{ }^{2}+\ldots
$$

where $g_{\alpha}$ is the linear coupling strength, $h_{\alpha}$ is the quadratic coupling strength, etc.

Having defined the Hamiltonian, we can proceed to the reduction of the Hamiltonian description of the motion to a map.

\section{The Classical Map}

The derivation of the map follows the standard procedure for reducing the Hamiltonian description of the motion to an iterated map. ${ }^{50-53}$ Instead of numerically solving the classical equations of motion in time, we will describe the dynamics of our system and bath by means of a difference equation. The set of difference equations, which specify the map, generates the evolution of the system and bath in discrete time intervals.

As mentioned previously, we are primarily interested in the energy exchange between the system and the bath in the weak coupling regime. Therefore, it is natural to use classical perturbation theory to obtain the map. Below we discuss the validity of this perturbation procedure and provide numerical as well as analytical arguments that support the use of perturbation theory.

Perturbation theory requires the use of a set of adiabatic variables, for which we use action-angle variables. It is well- 
known that the actions undergo relatively small changes in time, ${ }^{52}$ similar to the small mixing between different quantum states due to a perturbation, in quantum mechanics. There is one trivial nonclassical aspect of our use of action variables: we compute them in units of Planck's constant, so as to provide a direct correspondence with quantum numbers. However this is only a correspondence, and the actual dynamics follow the classical evolution equations. The system Hamiltonian, $h(q)$, and the bath Hamiltonian, $H_{\mathrm{b}}(\boldsymbol{Q})$, are chosen to be the zeroorder reference Hamiltonians and are rewritten for each system in terms of the action-angle variables. This is done separately for each system studied here, since the canonical transformation to action-angle variables depends on the system.

We now outline the steps required for the derivation of the map. The change in the action-angle variables is obtained by integrating Hamilton's equations of motion over time $\tau$ :

$$
\begin{gathered}
\Delta i=-\int_{0}^{\tau} \mathrm{d} t \frac{\partial H}{\partial \theta} \\
\Delta \theta=\int_{0}^{\tau} \mathrm{d} t \frac{\partial H}{\partial i}
\end{gathered}
$$

for the system mode, and

$$
\begin{gathered}
\Delta I_{\alpha}=-\int_{0}^{\tau} \mathrm{d} t \frac{\partial H}{\partial \Theta_{\alpha}} \\
\Delta \Theta_{\alpha}=\int_{0}^{\tau} \mathrm{d} t \frac{\partial H}{\partial I_{\alpha}}
\end{gathered}
$$

for the bath modes. In the above equations, $i$ and $\theta\left(I_{\alpha}\right.$ and $\Theta_{\alpha}$ ) are the system (bath mode $\alpha$ ) action and angle variables. The upper limit of the integral, $\tau$, is simply the time step of the integration. In molecular dynamics simulations this time step is chosen to be small enough so that the appropriate derivatives in eqs $9-12$ are replaced by their current values and can be taken out of the integral. ${ }^{63}$ In contrast, the map is obtained by extending the time step to values that are much larger than what current numerical integrators can handle. In fact, we are interested in time steps that are on the order of several vibrational periods of the system. Hence, instead of replacing the Hamiltonian derivatives in eqs $9-12$ by their current values, we approximate them using first-order classical perturbation theory. In other words, the perturbation is computed for the duration $\tau$ by using the current value of the action variables, while the angle variables vary with time according to the zeroorder Hamiltonian. The values of the action-angle variables are then updated, and first-order perturbation theory is used again, etc. [The current approach is somewhat similar to the so-called numerical analytical propagator algorithm (NAPA), which was developed by Tuckerman, Martyna, and Berne for systems with multiple time scales. ${ }^{64-67}$ Here, however, we are primarily interested in analytical corrections to the unperturbed motion, which are given numerically by NAPA.]

The above procedure is based on the assumption that during one integration step the action variables are varying slowly. This condition can be satisfied by properly choosing the integration step $\tau$. In the weak coupling regime, the time step can be taken to be as large as several vibrational periods of the system. For symmetry reasons we use a time step that is equal to an integer multiple of the system period (this time step will depend on the value of the system action). To zeroth order, the change in the system angle $\theta$ is equal to $2 \pi n$ (where $n$ is the number of vibrational periods in $\tau$ ). Therefore, we eliminate this variable from the equations by a simple transformation, which replaces the angles $\Theta_{\alpha}$ by the difference angles $\phi_{\alpha}$.

The advantage of the mapping procedure is that for the present set of systems we can analytically compute the changes in the action-angle variables for time $\tau$. This reduces the numerical effort needed to solve the classical equation of motion. Moreover, it enables a detailed examination of the integrated equation of motion, which can lead to further simplifications of the description of the motion. . $^{57,58}$

A. The Harmonic Oscillator. The harmonic oscillator has an analytic solution in the bilinear coupling limit (i.e., when only the first term in eq 8 is retained), so it is a natural system to test our method. In the action-angle variables the Hamiltonian take the form 60,68

$$
H=\omega i+\sum_{\alpha}^{N_{\mathrm{b}}} \Omega_{\alpha} I_{\alpha}+V(q, \mathbf{Q})
$$

where $i$ and $\omega\left(I_{\alpha}\right.$ and $\left.\Omega_{\alpha}\right)$ are the system (bath mode $\alpha$ ) vibrational action and frequency, respectively, and $V(q, \boldsymbol{Q})$ is given by eq 7. In terms of the action-angle variables, the massweighted coordinates $q$ and $Q_{\alpha}$ are given by

$$
q=\sqrt{(2 i / \omega)} \cos (\theta)
$$

and

$$
Q_{\alpha}=\sqrt{\left(2 I_{\alpha} / \Omega_{\alpha}\right)} \cos \left(\Theta_{\alpha}\right)
$$

To simplify the integrals in eqs 9 and 11, we note the relation between the time $t$ and the system vibrational angle variable $\theta$ $=\omega t$, to zeroth order in the coupling. This implies that $\theta$ can serve as a scaled time variable. The bath angle variables take a particularly simple form in zeroth order, which is also given in terms of the scaled time variables $\theta$ :

$$
\Theta_{\alpha}=\Omega_{\alpha} t+\phi_{\alpha}=\chi_{\alpha} \theta+\phi_{\alpha}
$$

where $\chi_{\alpha}=\Omega_{\alpha} / \omega$ is the ratio between the bath vibrational frequency of mode $\alpha$ and the system frequency, and $\phi_{\alpha}$ is introduced as a phase shift, which is the difference in the angle between the bath vibrational mode $\alpha$ and the system vibrational mode at the origin of time.

With these definitions, the map can readily be obtained. The changes in the action variables during $n$ periods of the system $(\tau=2 \pi n / \omega)$ are obtained by inserting eq 13 into eqs 9 and 11 . In terms of the scaled time variable, $\theta$, we find

$$
\begin{array}{r}
\Delta i=\frac{1}{\omega} \sum_{\alpha}^{N_{\mathrm{b}}}\left[g_{\alpha}^{*} \int_{-\pi n}^{\pi n} \mathrm{~d} \theta \sin (\theta) \cos \left(\chi_{\alpha} \theta+\phi_{\alpha}\right)+\right. \\
\left.\frac{h_{\mathrm{a}}^{*}}{2} \int_{-\pi n}^{\pi n} \mathrm{~d} \theta \sin (\theta) \cos ^{2}\left(\chi_{\alpha} \theta+\phi_{\alpha}\right)+\ldots\right]
\end{array}
$$

for the system, and

$$
\begin{aligned}
\Delta I_{\alpha} \frac{1}{\omega}\left[g_{\alpha}^{*} \int_{-\pi n}^{\pi n} \mathrm{~d} \theta \cos (\theta) \sin \left(\chi_{\alpha} \theta+\phi_{\alpha}\right)+\right. \\
\left.\frac{h_{\alpha}^{*}}{2} \int_{-\pi n}^{\pi n} \mathrm{~d} \theta \cos (\theta) \sin \left(2 \chi_{\alpha} \theta+2 \phi_{\alpha}\right)+\ldots\right]
\end{aligned}
$$

for the bath mode $\alpha$. In the above we have used an abbreviated 
notation for the scaled coupling constants $g_{\alpha}^{*}$ and $h_{\alpha}^{*}$, which depend on the current values of all action variables

$$
\begin{gathered}
g_{\alpha}^{*}=g_{\alpha}(2 i / \omega)^{1 / 2}\left(2 I_{\alpha} / \Omega_{\alpha}\right)^{1 / 2} \\
h_{\alpha}^{*}=h_{\alpha}(2 i / \omega)^{1 / 2}\left(2 I_{\alpha} / \Omega_{\alpha}\right)
\end{gathered}
$$

The integration over $\theta$ in eqs 17 and 18 can be done by using standard trigonometric relations ${ }^{69}$ and leads to the following explicit expressions for the changes in the system and bath actions during $n$ vibrational periods of the system:

$$
\begin{gathered}
\Delta i=\frac{\pi n}{\omega} \sum_{\alpha}^{N_{\mathrm{b}}}\left[g_{\alpha}^{*} f\left(\chi_{\alpha}\right) \sin \left(\phi_{\alpha}\right)+\frac{1}{4} h_{\alpha}^{*} f\left(2 \chi_{\alpha}\right) \sin \left(2 \phi_{\alpha}\right)+\ldots\right] \\
\Delta I_{\alpha}=\frac{\pi n}{\omega}\left[g_{\alpha}^{*} F\left(\chi_{\alpha}\right) \sin \left(\phi_{\alpha}\right)+\frac{1}{2} h_{\alpha}^{*} F\left(2 \chi_{\alpha}\right) \sin \left(2 \phi_{\alpha}\right)+\ldots\right]
\end{gathered}
$$

where the functions $f(x)$ and $F(x)$ are given by

$$
\begin{aligned}
& f(x)=\operatorname{sinc}((x+1) \pi n)-\operatorname{sinc}((x-1) \pi n) \\
& F(x)=\operatorname{sinc}((x+1) \pi n)+\operatorname{sinc}((x-1) \pi n)
\end{aligned}
$$

and we have used the notation $\operatorname{sinc}(x)=\sin (x) / x$ for the sinc function. ${ }^{70}$ To complete the specification of the map, we need the changes (per period) of the phase angles, $\phi_{\alpha}$. Since the first-order correction for $\phi_{\alpha}$ is relatively small, we use the change computed for an unperturbed motion, i.e., the zero-order change

$$
\Delta \phi_{\alpha}=2 \pi n \chi_{\alpha}
$$

It is interesting to compare the contribution to the changes in the action variables from the different term in eqs 20 and 21. We expect the magnitude of the coupling $g_{\alpha}^{*}$ and $h_{\alpha}^{*}$ to vary smoothly with $\alpha$ (see eq 54 below). Therefore, the role of the different bath modes will be governed by the sinc function, which is known to have a maximal value at the origin. ${ }^{70}$ This implies that the "resonant modes", i.e., modes that satisfy $\chi_{\alpha}=s^{-1}$, where $s$ is a positive integer, will have a dominant contribution to the dynamics of the system. It is also clear from eqs 20 and 21 that the first term in the Taylor expansion of the coupling sets a 1:1 resonance, while the second term sets a 1:2 resonance, etc.

Before we discuss some more realistic systems, let us examine the validity of the perturbation approximation. The change in the system action (given by eq 20) will provide a limit on the magnitude of the coupling strength, since it amounts to the sum of changes due to all bath modes. Hence we require that

$$
|\Delta i|<i
$$

for the perturbation method to be valid. We also carry out the treatment for the bilinear coupling only. The generalization to the complete coupling term can be done using similar arguments. In the bilinear coupling limit, the changes in the system action are given by

$$
\begin{aligned}
& \Delta i=\frac{\pi n}{\omega} \sum_{\alpha}^{N_{\mathrm{b}}} g_{\alpha}^{*} \sin \left(\phi_{\alpha}\right)\left(\operatorname{sinc}\left(\left(\chi_{\alpha}+1\right) \pi n\right)-\right. \\
&\left.\operatorname{sinc}\left(\left(\chi_{\alpha}-1\right) \pi n\right)\right)<i
\end{aligned}
$$

Since $\operatorname{sinc}\left(\left(\chi_{\alpha}-1\right) \pi n\right)>\operatorname{sinc}\left(\left(\chi_{\alpha}+1\right) \pi n\right)$ for each $\alpha$, we neglect the latter and replace $\operatorname{sinc}\left(\left(\chi_{\alpha}-1\right) \pi n\right) \sin \left(\phi_{\alpha}\right)$ by its maximal value of 1 for each mode. This clearly overestimates the magnitude of the change in $i$, since not all bath modes are in phase with the system oscillator and not all of them are in a $1: 1$ resonance. In the limit of equal "kicks" from all bath modes we have

$$
\mathrm{g}_{\alpha}^{*}<\frac{\omega i}{n \pi N_{\mathrm{b}}}
$$

A numerical illustration of the accuracy of the maps is given in section $\mathrm{V}$ for the harmonic oscillator and the other two systems studied here. Note that for small energies, i.e., when $i \rightarrow 0$, the present approach breaks down, because eq 26 can no longer be satisfied. The present approach will also break down for high energies near the dissociation in the Morse oscillator studied below.

B. The Morse Oscillator. We now turn to the Morse oscillator, which is a more realistic model for molecular systems. The map for this system is similar to the harmonic map, even though the two Hamiltonians differ significantly. The Hamiltonian in action-angle variables takes the form ${ }^{60}$

$$
H=\beta(2 D)^{1 / 2} i-\frac{1}{2} \beta^{2} i^{2}+\sum_{\alpha}^{N_{\mathrm{b}}} \Omega_{\alpha} I_{\alpha}+V(q, \mathbf{Q})
$$

where $D$ and $\beta$ are the well depth and the mass-weighted range parameters of the Morse potential, respectively. To obtain the proper harmonic limit, we modify the coupling $V(q, \boldsymbol{Q})$ between the Morse oscillator and the bath. Specifically we replace eq 7 by

$$
V(q, \mathbf{Q})=-\left(1-\mathrm{e}^{\beta q}\right) f(\mathbf{Q}) / \beta
$$

which is nonlinear in both the system and bath displacement and reduces to eq 7 for small values of $q(f(\boldsymbol{Q})$ is given by eq $8)$. In terms of the action-angle variables $i$ and $\theta$, the massweighted coordinate $q$ is given by ${ }^{60}$

$$
q=\beta^{-1} \ln \left(\frac{1+\sqrt{\epsilon(i)} \cos (\theta)}{1-\epsilon(i)}\right)
$$

where $\epsilon$ is the Morse anharmonicity parameter

$$
\epsilon(i)=\beta(2 / D)^{1 / 2} i-\frac{1}{2 D} \beta^{2} i^{2}
$$

The changes in the action variables during $n$ vibrational periods of the system $(\tau=2 \pi n / \omega(i))$ are obtained following similar steps taken to derive the harmonic map. Note that for the Morse oscillator the time step $\tau$ depends on the current value of the system action. Thus we do not use a constant time step when integrating eqs 20 and 21, but rather change the time step between each update of the actions. This leads to the following 
set of equations for the changes in the action variables during $n$ vibrational periods:

$$
\begin{aligned}
& \Delta i= \\
& \quad \frac{\pi n}{\omega(i)} \sum_{\alpha}^{N_{\mathrm{b}}}\left[g_{\alpha}^{*} f\left(\chi_{\alpha}(i)\right) \sin \left(\phi_{\alpha}\right)+\frac{1}{4} h_{\alpha}^{*} f\left(2 \chi_{\alpha}(i)\right) \sin \left(2 \phi_{\alpha}\right)+\ldots\right]
\end{aligned}
$$

$$
\begin{aligned}
& \Delta I_{\alpha}= \\
& \frac{\pi n}{\omega(i)}\left[g_{\alpha}^{*} F\left(\chi_{\alpha}(i)\right) \sin \left(\phi_{\alpha}\right)+\frac{1}{?} h_{\alpha}^{*} F\left(2 \chi_{\alpha}(i)\right) \sin \left(2 \phi_{\alpha}\right)+\ldots\right]
\end{aligned}
$$

where for the Morse potential the functions $f(x)$ and $F(x)$ take the form

$$
\begin{gathered}
f(x)=\operatorname{sinc}((x+1) \pi n)-\operatorname{sinc}((x-1) \pi n) \\
F(x)=\operatorname{sinc}((x+1) \pi n)+\operatorname{sinc}((x-1) \pi n)+ \\
2 \sqrt{\epsilon(i)} \operatorname{sinc}(\pi n x)
\end{gathered}
$$

In the above equations $\omega(i)=\beta(2 D)^{1 / 2}-\beta^{2} i$ is the actiondependent vibrational frequency of the Morse system, and $\chi_{\alpha}(i)$ $=\Omega_{\alpha} / \omega(i)$ is the ratio between the bath vibrational frequency of mode $\alpha$ and the system frequency. Unlike the harmonic case, this ratio depends on the current value of the vibrational action $i$. The scaled coupling constants take a slightly different form:

$$
\begin{gathered}
g_{\alpha}^{*}=g_{\alpha} \sqrt{\epsilon(i)}\left(2 I_{\alpha} / \Omega_{\alpha}\right)^{1 / 2} /((1-\epsilon(i)) \beta) \\
h_{\alpha}^{*}=\mathrm{h}_{\alpha} \sqrt{\epsilon(i)}\left(2 I_{\alpha} / \Omega_{\alpha}\right) /((1-\epsilon(i)) \beta)
\end{gathered}
$$

The map is completed by specifying the change, during $n$ periods, in the phase angles $\phi_{\alpha}$, which is given for an unperturbed motion by

$$
\Delta \phi_{\alpha}=2 \pi n \chi_{\alpha}(i)
$$

The harmonic limit of the Morse map is obtained by carefully taking the appropriate limit of the Morse potential parameters. Specifically, when $\beta \rightarrow 0$ and $D \rightarrow \infty$ so that the product $\beta \sqrt{D}$ is constant, eqs 31 and 32 reduce to eqs 20 and 21 , respectively.

C. The Double Well Potential. We now turn to the map for the double-well potential. Unlike the previous two systems, an exact transformation to action-angle variables for the doublewell potential does not exist. Therefore, we derive approximate action-angle variables using a procedure similar to one suggested by Born. ${ }^{50}$ The details of the transformation can be found in the Appendix. The Hamiltonian in action-angle variables takes the form

$$
H=E(i)+\sum_{\alpha}^{N_{\mathrm{b}}} \Omega_{\alpha} I_{\alpha}+V(q, \mathbf{Q})
$$

The energy of the double-well potential, $E(i)$, is given in the Appendix. The coupling, $V(q, \boldsymbol{Q})$, is given by eq 7 , and the mass-weighted coordinate $q$ is defined in the Appendix (cf. eq A12).

With these definitions, the map can be obtained following the procedure outlined in the previous subsection. We note in passing that the time step $\tau=2 \pi n / \omega(i)$ depends on the current value of the action, just like in the Morse oscillator. Performing the mapping transformation, we find that the changes in the action-angle variables during $n$ periods of the system are given by

$$
\begin{aligned}
& \Delta i= \\
& \quad \frac{\pi n}{\omega(i)} \sum_{\alpha}^{N_{\mathrm{b}}}\left[g_{\alpha}^{*} f\left(\chi_{\alpha}(i)\right) \sin \left(\phi_{\alpha}\right)+\frac{1}{4} h_{\alpha}^{*} f\left(2 \chi_{\alpha}(i)\right) \sin \left(2 \phi_{\alpha}\right)+\ldots\right]
\end{aligned}
$$

for the system action,

$$
\begin{aligned}
& \Delta I_{\alpha}= \\
& \frac{\pi n}{\omega(i)}\left[g_{\alpha}^{*} F\left(\chi_{\alpha}(i)\right) \sin \left(\phi_{\alpha}\right)+\frac{1}{2} h_{\alpha}^{*} F\left(2 \chi_{\alpha}(i)\right) \sin \left(2 \phi_{\alpha}\right)+\ldots\right]
\end{aligned}
$$

for the bath actions, and

$$
\Delta \phi_{\alpha}=2 \pi n \chi_{\alpha}(i)
$$

for the change in the angle variables. The latter is given for the unperturbed motion, i.e., the zero-order change. The functions $f(x)$ and $F(x)$ for the double-well potential take a different form:

$$
\begin{gathered}
f(x)=J_{x}^{\prime}(\epsilon x) \\
F(x)=J_{x}^{\prime}(\epsilon x) / x+\left((-)^{n}-\epsilon\right) \operatorname{sinc}(\pi n x)
\end{gathered}
$$

where $J_{x}^{\prime}(\epsilon x)$ denotes the derivative of the Anger function with respect to its argument, ${ }^{71}$

$J_{x}^{\prime}(\epsilon x)=\frac{1}{2 \pi} \int_{-\pi}^{\pi} \mathrm{d} \psi \sin (n \psi) \sin [x(n \psi-\epsilon \sin (n \psi))]$

For our purposes, it is sufficient to expand the derivative of the Anger function in a power series in $\epsilon \approx\left(e_{1}+e_{2}\right) /\left(e_{1}-\right.$ $\left.e_{2}\right)$, where $e_{1}$ and $e_{2}$ are the roots of eq A4. Recall that one of the roots is negative, so that $\epsilon$ is a direct measure of the anharmonicity. Retaining only terms up to linear order in $\epsilon$, we find

$$
\begin{aligned}
& J_{x}^{\prime}(\epsilon x) \approx \frac{1}{2}[\operatorname{sinc}((x-1) \pi n)-\operatorname{sinc}((x+1) \pi n)]+ \\
& \quad \frac{\epsilon}{4}[\operatorname{sinc}((x+2) \pi n)+\operatorname{sinc}((x-2) \pi n)-2 \sin (\pi n x) /(\pi n)]
\end{aligned}
$$

This approximation is consistent with the perturbative approach taken in this paper and is numerically found to be realistic for $\epsilon<0.2$ (for $n=1$ ).

In the above equations $\omega(i)=\partial \mathrm{E}(i) / \partial i$ is the system frequency, and $\chi_{\alpha}(i)=\Omega_{\alpha} / \omega(i)$ is the bath to system frequency ratio. The scaled coupling constants are now expressed in terms of the roots $e_{1}$ and $e_{2}$ :

$$
\begin{gathered}
g_{\alpha}^{*}=g_{\alpha}\left(e_{1}-e_{2}\right)\left(2 I_{\alpha} / \Omega_{\alpha}\right)^{1 / 2} \\
h_{\alpha}^{*}=\mathrm{h}_{\alpha}\left(e_{1}-e_{2}\right)\left(2 I_{\alpha} / \Omega_{\alpha}\right)
\end{gathered}
$$

In the limit where $\epsilon \rightarrow 0$, eqs 37 and 38 reduce to the harmonic map given by eqs 20 and 21 . This can be seen with the help of the identities

$$
\begin{array}{r}
\operatorname{sinc}((x+1) \pi n)+\operatorname{sinc}((x-1) \pi n)=\frac{1}{x}[\operatorname{sinc}((x-1) \pi n)- \\
\left.\operatorname{sinc}((x+1) \pi n)+(-)^{n} 2 \sin (\pi n x) /(\pi n)\right]
\end{array}
$$


and

$$
e_{1}-e_{2}=-2 \sqrt{2 i / \omega}
$$

For finite anharmonicities, the map specified in eqs 37 and 38 is slightly different from the harmonic and Morse maps. The main difference can be traced to higher order resonances in the system mode, which arise from the more complicated dynamics in the double-well potential (i.e., there are higher harmonics in the form of the coupling between the system and the bath). These higher harmonics enter the map through the second term in eq 42.

\section{The Fokker-Planck Equation}

In this section we introduce a random phase approximation, ${ }^{57}$ which will provide the grounds to further simplify the description of the motion. In this approximation, the maps can immediately be reduced to a Fokker-Planck equation in action space. However, this simplification is not trivial, and even simple maps can give rise to quite complex evolution in action space because of the dependence on the initial conditions.

The reduction of the maps to a Fokker-Planck equation is not only an alternative way of describing the dynamics of the system but rather a way of simplifying this description. The reduction is based on the adiabatic nature of the action variables and on the relatively rapid changes in the angle variables. In the kinetic limit, the dynamics can be described by ${ }^{52}$

$$
\partial_{\tau} P=-\sum_{\alpha}^{N_{\mathrm{b}}+1} \partial_{J_{\alpha}}\left(A_{\alpha} P\right)+\frac{1}{2} \sum_{\alpha, \beta}^{N_{\mathrm{b}}+1} \partial_{J_{\alpha}} \partial_{J_{\beta}}\left(B_{\alpha, \beta} P\right)
$$

where $\partial_{J_{\alpha}} \equiv \partial / \partial J_{\alpha}$, and we use the notation $J_{1} \equiv i$ and $J_{\alpha \neq 1} \equiv$ $I_{\alpha}$. The coefficients $A_{\alpha}$ and $B_{\alpha, \beta}$ are given by

$$
A_{1}=\langle\Delta i\rangle, \quad A_{\alpha \neq 1}=\left\langle\Delta I_{\alpha}\right\rangle
$$

and

$$
\begin{array}{r}
B_{1,1}=\left\langle(\Delta i)^{2}\right\rangle, \quad B_{\alpha \neq 1, \beta \neq 1}\left\langle\Delta I_{\alpha} \Delta I_{\beta}\right\rangle, \\
B_{1, \alpha \neq 1}=B_{\alpha \neq 1,1}\left\langle\Delta i \Delta I_{\alpha}\right\rangle
\end{array}
$$

The brackets, $\langle\ldots\rangle$, denote a particular way of averaging over time. In the random phase approximation this average can be taken as an average over the initial phases, which are uniformly distributed between 0 and $2 \pi$. In other words, the averaging over time is replaced by an average over the initial phases due to the ergodicity of the angles. The assumption made is that the changes in the phases are not correlated from one round to the next. We note that it is possible to include correlations between different rounds in a simple, yet ad hoc, way. ${ }^{52}$

In the random phase approximation, eq 46 can be reduced to the so-called divergence form ${ }^{52}$

$$
\partial \tau P=\frac{1}{2} \sum_{\alpha, \beta}^{N_{\mathrm{b}}+1} \partial_{J_{\alpha}}\left(D_{\alpha, \beta} \partial_{J_{\beta}} P\right)
$$

where $D_{\alpha, \beta}$ are the diffusion coefficients. This form of the Fokker-Planck equation can be obtained with the help of the relation $A_{\alpha}=(1 / 2) \partial_{J_{\beta}} B_{\alpha, \beta}$, which was first derived by Landau. ${ }^{72}$ We note that this relation holds only in the random phase approximation, which assumes a uniform distribution of the angles. For real systems this is never the case, and therefore the random phase limit is but an approximation.
The regime in which the random phase approximation is valid for the current maps is obtained from an explicit calculation of the evolution of the phases. The latter is given by $\Delta \phi_{\alpha}=$ $2 \pi n \chi_{\alpha}(i)$, for the unperturbed motion. In the random phase approximation, it is assumed that the phase $\phi_{\alpha}$ varies so much between one round to the next that $\sin \left(\phi_{\alpha}\right)$ can be regarded as a random variable. The analytical justification for this approximation is that, in the limit $n \gg 1$, the change in the phases is indeed very large. In some of the applications reported in this paper we have used $n=20$, in which the random phase limit is shown to be adequate.

Based on the uniform distribution of the angles, the diffusion coefficients $D_{\alpha, \beta}$ are given by

$$
\begin{aligned}
& D_{1,1}\left(\frac{\pi n}{2 \omega(i)}\right) \sum_{\alpha}^{N_{\mathrm{b}}}\left\{\left(g_{\alpha}^{*}\right)^{2} f\left(\chi_{\alpha}(i)\right)^{2}+\frac{1}{16}\left(h_{\alpha}^{*}\right)^{2} f\left(2 \chi_{\alpha}(i)\right) 2\right\} \\
& D_{\alpha, \alpha \neq 1}=\left(\frac{\pi n}{2 \omega(i)}\right)\left\{\left(g_{\alpha}^{*}\right)^{2} F\left(\chi_{\alpha}(i)\right)^{2}+\frac{1}{4}\left(h_{\alpha}^{*}\right)^{2} F\left(2 \chi_{\alpha}(i)\right)^{2}\right\} \\
& D_{1, \alpha \neq 1}=\left(\frac{\pi n}{2 \omega(i)}\right)\left\{\left(g_{\alpha}^{*}\right)^{2} f\left(\chi_{\alpha}(i)\right) F\left(\chi_{\alpha}(i)\right)+\right. \\
& \left.\frac{1}{8}\left(h_{\alpha}^{*}\right)^{2} f\left(2 \chi_{\alpha}(i)\right) F\left(2 \chi_{\alpha}(i)\right)\right\}
\end{aligned}
$$

and all the other diffusion coefficients equal zero. $f(x)$ and $F(x)$ are given in section III for the three systems studied here.

Equations 50-52 are the central results of this section. In the random phase approximation, the ensemble-averaged motion is diffusive, with no drift term. The diffusion coefficients depend on the current value of all action variables and are analytically given in the above equations. We note that it is also possible to reduce the maps to a one-dimensional FokkerPlanck equation in the system action only. The procedure can be found in ref 21 and will not be repeated here.

The solution of the Fokker-Planck equation is not trivial due to the fact that the diffusion coefficients depend on the actions. ${ }^{61,62}$ The evolution in action space can be obtained by adopting a semiclassical approach ${ }^{73}$ or using a numerical procedure. ${ }^{74}$ Alternatively, one can obtain an approximate solution using a Gaussian ansatz for the distribution. ${ }^{75}$

Before we present the results, we outline the differences between the current approach and other approaches to the problem. Based on the Langevin equation, Kramers derives a one-dimensional Fokker-Planck equation for his chemical reaction model in the low-viscosity limit, ${ }^{1,20}$ and others ${ }^{9,11,14,40}$ have extended Kramers' approach for non-Markovian processes by using a generalized Langevin equation. ${ }^{39}$ Both methods assume a Gaussian random fluctuating force associated with the thermal bath. The latter can be derived starting from a Hamiltonian, in which the bath is described by a collection of harmonic modes and the coupling is linear in the bath modes. ${ }^{41}$ Tuckerman and Berne showed that this simple model can describe the energy relaxation in a particular highly nonlinear system and bath. ${ }^{35}$ The present approach differs from previous studies with respect to two major points: we explicitly take the bath into account and therefore can handle nonlinear couplings (even in the system mode), and we treat the kinetic limit with a multidimensional Fokker-Planck equation in action space.

Using the current results, it is possible to obtain the rate of escape over the barrier in the double-well model ${ }^{20}$ and the mean first passage time for energy accumulation in the two different system oscillators. ${ }^{40}$ The derivation of these two important 
quantities is left open for future study. Here instead we focus on assessing the validity of the mapping procedure for energy accumulation and dissipation.

\section{Results}

In this section we assess the accuracy of the mapping procedure. The maps are solved numerically, and the results are compared with the Hamiltonian dynamics for individual trajectories as well as for an ensemble of trajectories. To perform the calculation we represent the continuous bath by a discretized sum of harmonic modes. We follow a procedure which is slightly different from the standard one $;{ }^{76}$ namely, the bath modes are generated from the density

$$
\rho(\omega)=2 \gamma \omega \exp \left(-\gamma \omega^{2}\right)
$$

where $\gamma=1.2 \times 10^{7}$. From now on we employ atomic units. The linear dependence for small frequencies is consistent with localized behavior of soft modes ${ }^{77}$ and has been observed in Lennard-Jones liquids. ${ }^{78,79}$

To assess the accuracy of the map, we retain only the leading term in the system-bath coupling, i.e., the sum of bilinear terms. The coupling between the system and the bath due to the quadratic and higher order terms is typically smaller than the coupling due to the bilinear term. In particular, those terms are not expected to contribute significantly to energy dissipation when the system frequency is well inside the relevant range of the spectral density (this is the case for all the systems studied below). ${ }^{80}$ Therefore, we focus only on the simpler case of bilinear system-bath coupling and leave the assessment of the validity of the map when higher order terms are included for future study. ${ }^{81}$

In the present case, the linear coupling coefficients were calculated according to

$$
g_{\alpha}{ }^{2}=\left(J\left(\omega_{\alpha}\right) / \rho\left(\omega_{\alpha}\right)\right) \delta \omega=2 \lambda \sqrt{\frac{\gamma}{\pi}} \omega_{\alpha} \delta \omega
$$

where $\delta \omega=\omega_{\max } / N_{\mathrm{b}}, \omega_{\max }$ is a cutoff frequency introduced for numerical reasons, and $J(\omega)$ is the spectral density. For all the results shown in this section we use a cutoff $\omega_{\max }=9 \times 10^{-4}$ in atomic units. Within the deformation potential approximation, ${ }^{76,82}$ the spectral density takes the form

$$
J(\omega)=4 \lambda \gamma \sqrt{\frac{\gamma}{\pi}} \omega^{2} \exp \left(-\gamma \omega^{2}\right)
$$

which is proportional to $\omega^{2}$ for small frequencies, i.e., a superohmic spectral density. $\lambda$ is the overall system-bath coupling strength. We note that when necessary, the higher order coupling terms can be obtained by following the procedure suggested by Egorov and Berne. ${ }^{59}$

It is rather straightforward to generate trajectories using the map. Given the current value of the actions and the phases, one computes the value of the change and updates the actions and angles. This procedure is repeated until the desired time is reached. The physical time is increased by one period $(\tau)$ from one round to the next. To compute the actual change in the actions, we use a predictor-corrector algorithm, which is essential to ensure numerical stability of the map. In the first step, we predict the change in the action variables using the current value of the actions. The second step corrects the change in the action variables given their predicted value. The procedure is required since the maps were obtained by firstorder perturbation theory, and therefore they do not preserve

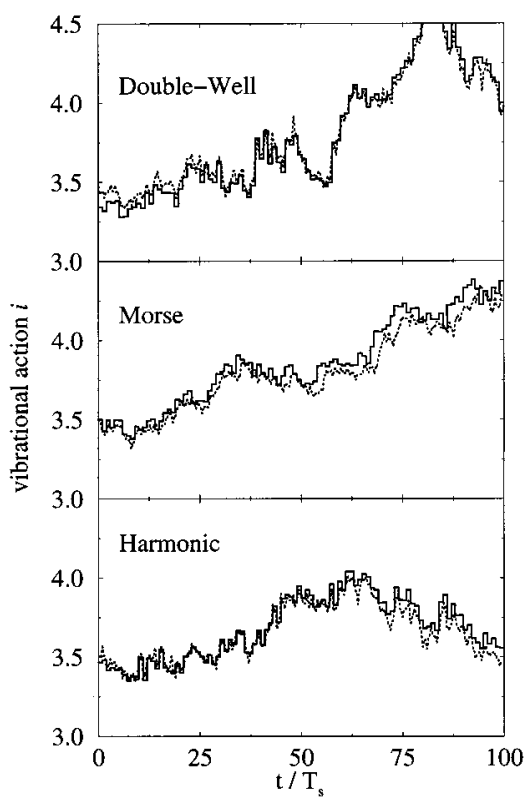

Figure 1. Plots of typical trajectories for the harmonic, Morse, and double-well systems. Shown are the changes in the system action, $i$, vs the reduced time $t / T_{\mathrm{s}}$, where $T_{\mathrm{s}}$ is the system period at the origin of time. The following parameters are used: $\omega=5.4 \times 10^{-4}$ for the harmonic oscillator; $D=0.04$ and $\beta=0.61$ for the Morse oscillator; $k=0.03, c=-0.01$, and $d=1.5 \times 10^{-3}$ for the double-well potential; the coupling strength $\lambda=1.1 \times 10^{-11}$ and the reduce mass is $m=$ 115570 for all three cases. The solid and dashed lines are the results for the mapped and Hamiltonian trajectories, respectively. Note the excellent agreement between the current approach and the Hamiltonian dynamics.

phase space volume (the maps were not derived from a generating function). Our predictor-corrector method is thus equivalent to symplectic integrators used in classical molecular dynamics simulations. ${ }^{63}$

To compare individual trajectories, we set the initial action and angle variables of the Hamiltonian dynamics to be equal to the initial actions and phases of the map, respectively. The latter are chosen from a Maxwell-Boltzmann distribution, excluding the system action which takes a sharp value at the origin of time. This implies that the angles are selected from a random distribution between 0 and $2 \pi$, and the actions are generated from $\exp \left(-\Omega_{\alpha} I_{\alpha} / k_{\mathrm{b}} T\right) / Q$, where $T$ is the temperature $\left(T=3.17 \times 10^{-3}\right.$ au for all results shown here) and $Q$ is the normalization of the distribution function. This procedure amounts to a factorization of the initial distribution, that is, in the spirit of the perturbation approximation we neglect the coupling between the system and the bath for the initial conditions. We note that this approximation is not required and is introduced for simplicity.

In Figures 1-3 we show typical trajectories for the three systems under study. The mapped trajectories are characterized by discontinuous jumps in their actions, because we compute the action at large time intervals. In Figures 1 and 2 we use relatively large coupling coefficients $\left(\lambda=1.1 \times 10^{-11}\right)$, and to ensure an adequate description of the map, the time step is set to one vibrational period of the system, i.e., $n=1$. The results shown in Figure 3 are for a smaller overall systembath coupling strength, $\lambda=3.5 \times 10^{-12}$, so that the time step is set to 20 vibrational periods of the system, i.e., $n=20$. With these choices of the time step, the mapped dynamics require $20 \%$ and $1 \%$ the CPU time required for the Hamiltonian dynamics (which are much more accurate), respectively, and for an arbitrary time step we find that the ratio is about $1: 5 n$. 


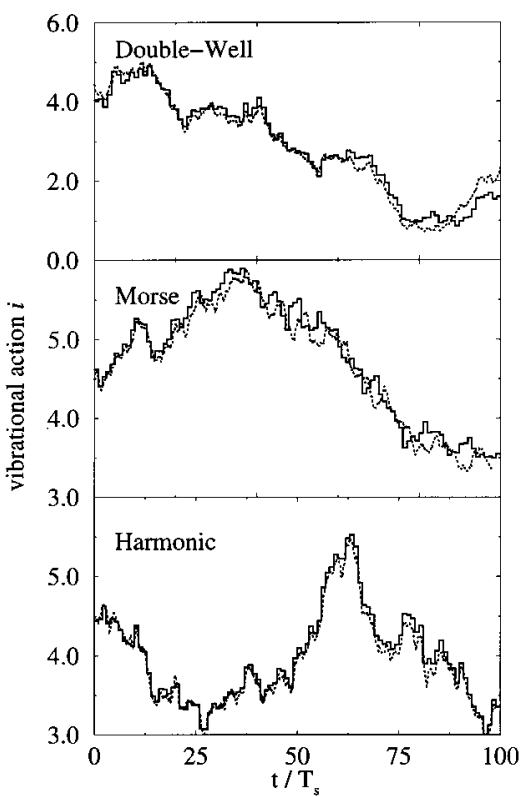

Figure 2. The same as Figure 1 but for a different set of system parameters: $\omega=3.1 \times 10^{-4}$ for the harmonic oscillator; $D=0.02$ and $\beta=0.43$ for the Morse oscillator; $k=0.01, c=-0.003$, and $d=$ $4.5 \times 10^{-4}$ for the double-well potential.

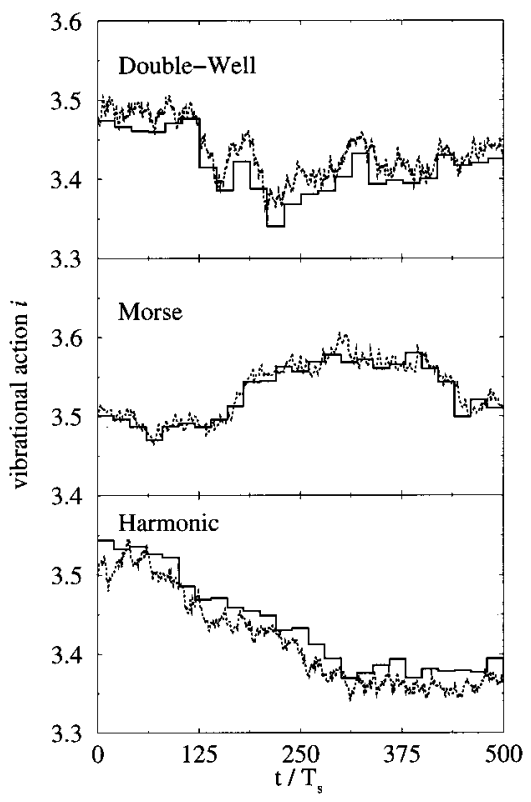

Figure 3. The same as Figure 1 but for a different coupling strength, $\lambda=3.5 \times 10^{-12}$. This coupling strength induces much smaller energy exchanges between the system and the bath, and therefore we use a much larger integration step for the map $(n=20)$.

Since the map is only an approximation to the true dynamics, a real systematic comparison of CPU time with molecular dynamics is not possible. The reason is that the energy conservation in the map, though very stable, is at a level for which ordinary integration schemes, such as the Verlet integrator, explode. Since a speedup of the CPU time is not the major goal of the mapping procedure, we do not elaborate on this point any further.

It is clearly seen in Figures $1-3$ that the map provides a realistic description of the dynamics for all three systems. The trajectories are in excellent agreement with the exact Hamiltonian dynamics. Note the small changes in the system action during one iteration, which is a necessary condition for the mapping procedure to work. We have chosen the coupling

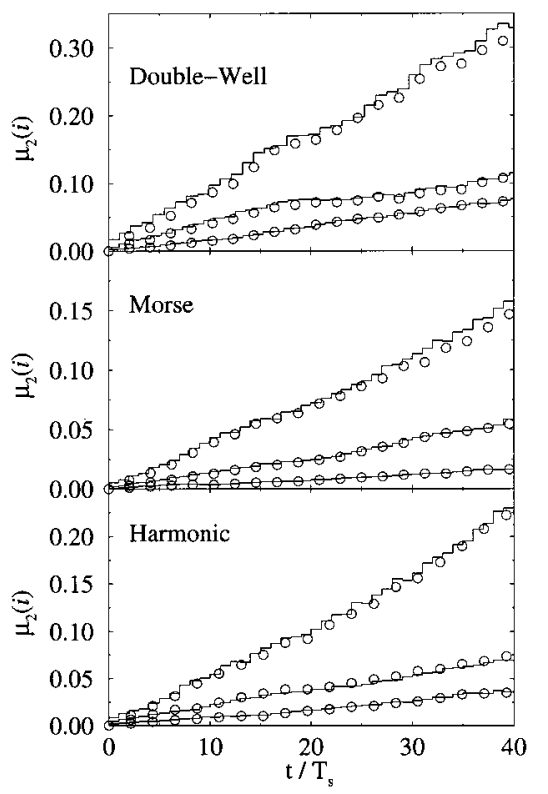

Figure 4. Plots of the second moment of the system action vs reduced time $t / T_{\mathrm{s}}$, where $T_{\mathrm{s}}$ is the system period at the origin of time. The lines and symbols are the results of the maps and Hamiltonian dynamics, respectively. The results are averaged over 300 trajectories and $N_{\mathrm{b}}=$ 1000. The initial system actions are 1.5, 3.5, and 6.5 in increasing slope order. Similar agreement is found for the first moment too.

parameters to provide significant changes in the action over the time scale shown in Figures 1 and 2. In many realistic systems, this time is much longer and requires many iteration of the map. In such cases, the natural time step $(\tau)$ used to obtain the map can be much larger than the vibrational period of the system, but not so large that the changes in the system action during one iteration exceed the value of the action. This is exactly the case shown in Figure 3, in which the time step of the map is increased to 20 vibrational periods.

We find that the agreement between the map and the Hamiltonian dynamics is excellent for a large range of initial conditions and different sets of parameters and couplings. The map provides better results at lower energies due to the energy scaling of the system-bath coupling. We do, however, find some trajectories that deviate slightly from the Hamiltonian dynamics. These are only a small fraction of the total number of trajectories used to average over initial conditions, and therefore they have a minor effect on averaged properties.

The results for an ensemble of trajectories are shown in Figure 4. We plot the second moment of the system action $\left(\mu_{2}=\left\langle i^{2}\right\rangle\right.$ $-\langle i\rangle^{2}$, where $\langle\ldots\rangle$ denote an ensemble average) versus the reduced time for all three systems. The agreement between the mapped and Hamiltonian ensembles is excellent for a wide range of initial energies. We note that a similar agreement is obtained for the first moment of the system action, which is related to the energy relaxation.

In Figure 5 we compare the analytical results for the second moment in the system action with results obtained by numerically iterating the maps. It is clear that for the present coupling regime the random phase approximation adequately describes the dynamics of the system, so that the kinetic description given by the Fokker-Planck equation is valid. The harmonic system displays a linear $i$ dependence of this second moment, while both the Morse and double-well systems deviate from linearity at large values of $i$. This behavior can be traced to the fact that the frequency, $\omega$, is independent of the action $i$ for the harmonic system. 


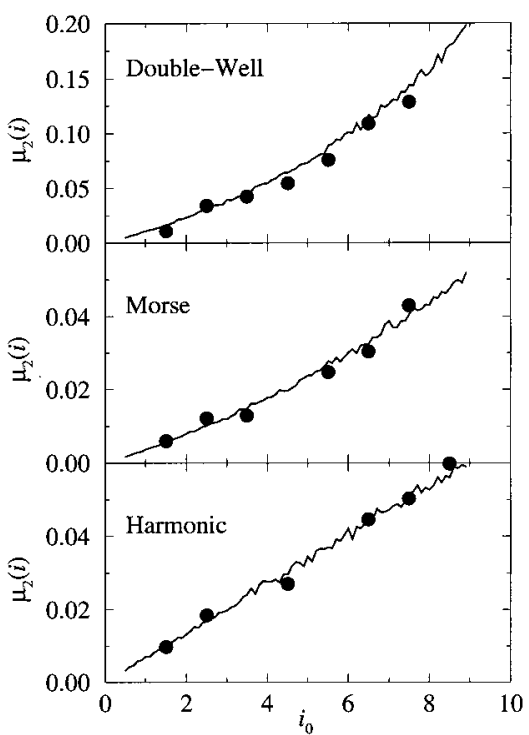

Figure 5. Plots of the second moment of the system action vs its initial value $i_{0}$. The lines are the results of the the analytical formula given by eqs $50-52$, and the symbols numerical solution of the maps. The numerical results are averaged over 1000 trajectories and $N_{\mathrm{b}}=10000$. The number of vibrational periods in one time step is $n=20$. Note the nonlinear dependence of $\mu_{2}(i)$ for the Morse and double-well systems.

\section{Concluding Remarks}

We have shown that the dynamics of a system coupled to a bath can be captured by a simple map. The map provides a realistic approximation to the dynamics, not only for averaged properties but also for individual trajectories, and it works equally well for linear and nonlinear systems. The map is not only is an efficient way to simulate the dynamics of the system but also provides a simplified description for the different Hamiltonians, and it highlights the various coupling parameters that govern the motion of the system.

In the random phase approximation, the maps can be reduced to the Fokker-Planck equation. This kinetic description is not only an alternative way of describing the dynamics of the system but rather a way of simplifying this description and providing useful insights into the problem. We have derived explicit analytic expressions for the energy-diffusion coefficients in terms of the various parameters (coupling strength, frequency, initial energy, etc.).

The treatment of higher order couplings which is required for more realistic systems, the solution of the multidimensional Fokker-Planck equation, and the application of the mapping method to calculate rate constants are still open problems for future study.

Acknowledgment. E.R. is delighted to thank Raphy Levine for introducing him to the world of nonlinear dynamics. He is also grateful to Joseph Klafter for insightful comments, discussions, and encouragement. Sergei Egorov, Dan Gezelter, and Philip Pechukas are thanked for several discussions and helpful comments on the manuscript. The Rothschild and Fulbright foundations are acknowledged for financial support to E.R. This work was supported in part by a grant to B.J.B. from the National Science Foundation.

\section{Appendix A: Approximate Action-Angle Variables for the Double-Well Potential}

In this Appendix we derive approximate action-angle variables for the double-well potential in the form of an expansion in powers of the small anharmonic terms in the potential. We seek an expression of the energy of the doublewell in terms of the action variable $i$ and for a relation between the displacement $q$ and the action-angle variables. The action variable $i$ is defined by

$$
i=\frac{1}{2 \pi} \oint p d q=\sqrt{2 d e_{3} e_{4}}\left(i_{0}-\frac{1}{2 \mu} i_{1}-\frac{1}{8 \mu^{2}} i_{2}+\ldots\right)
$$

where

$$
\begin{gathered}
i_{j}=\frac{1}{2 \pi} \oint q^{j} \sqrt{\left(e_{1}-q\right)\left(q-e_{2}\right)} d q \\
\frac{1}{\mu}=\frac{1}{e_{3}}+\frac{1}{e_{4}}
\end{gathered}
$$

and the $e_{j}^{\prime}$ 's are the roots (in ascending order, $\left|e_{1}\right|,\left|e_{2}\right|<e_{3}<$ $e_{4}$ ) of

$$
\begin{aligned}
& g(q)=\frac{E}{d}-\frac{1}{2 d} \omega^{2} q^{2}-\frac{c}{d} q^{3}-q^{4}= \\
& \quad e_{3} e_{4}\left(e_{1}-q\right)\left(q-e_{2}\right)\left(1-\frac{q}{e_{3}}\right)\left(1-\frac{q}{e_{4}}\right)
\end{aligned}
$$

The integration of eq A2 can be done by means of the substitution

$$
q=\frac{1}{2}\left(e_{1}-e_{2}\right) \sin \psi+\frac{1}{2}\left(e_{1}+e_{2}\right)
$$

and leads to an explicit expression for the actions $i_{j}$ in terms of the roots $e_{1}$ and $e_{2}$ :

$$
\begin{gathered}
i_{0}=\frac{1}{8}\left(e_{1}-e_{2}\right)^{2} \\
i_{1}=\frac{1}{2}\left(e_{1}+e_{2}\right) i_{0} \\
i_{2}=\frac{1}{16}\left[5\left(e_{1}+e_{2}\right)^{2}-4 e_{1} e_{2}\right] i_{0}
\end{gathered}
$$

The roots, $e_{j}$, can be obtained by expanding them in a power series in $d$, or alternatively by adopting a self-consistent approach, such as the Newton-Raphson method. ${ }^{83}$ Since the roots depend on the system energy $E$, we have $i(E)$ and also $E(i)$ by inverting eq A1. This is one of the goals set for this Appendix.

Next we calculate $q$ in terms of the action-angle variables $i$ and $\theta$. To the order required, the angle is given by

$$
\theta \sqrt{\frac{1}{2 d e_{3} e_{4}}} \omega(i)\left(K_{0}+\frac{1}{2 \mu} K_{1}\right)=\kappa \psi-\epsilon \cos (\psi)
$$

where the $K_{j}^{\prime}$ 's are obtained using A5:

$$
\begin{gathered}
K_{0}=\psi \\
K_{1}=\frac{1}{2}\left(e_{1}+e_{2}\right) \psi-\frac{1}{2}\left(e_{1}-e_{2}\right) \cos (\psi)
\end{gathered}
$$

In the above equations $\omega(i)=\partial \mathrm{E}(i) / \partial i$ is the action-dependent frequency,

$$
\kappa \sqrt{\frac{1}{2 d e_{3} e_{4}}} \omega(i)\left(1+\frac{e_{1}+e_{2}}{4 \mu}\right) \approx 1
$$


and

$$
\epsilon=\sqrt{\frac{1}{2 d e_{3} e_{4}}} \omega(i)\left(1+\frac{e_{1}-e_{2}}{4 \mu}\right) \approx \frac{e_{1}+e_{2}}{e_{1}-e_{2}}
$$

The approximations for $\kappa$ and for $\epsilon$ in eqs A9 and A10 can be obtained from an expansion of the roots in a power series in $d$.

Finally, we set the origin of the angle $\psi$ to be on the inner turning point (which is $e_{1}$ ), so that the angle variable takes the form

$$
\theta \approx \psi-\epsilon \sin (\psi)
$$

and the displacement $q$ is

$$
q \approx \frac{1}{2}\left(e_{2}-e_{1}\right) \cos (\psi)+\frac{1}{2}\left(e_{1}+e_{2}\right)
$$

We have compared this analytic solution with trajectories obtained by an exact numerical solution of Hamilton's equations of motion for the one-dimensional double-well potential. The agreement is found to be excellent, even for energies that are very close to the barrier. Note that the agreement depends on the values of the roots $e_{3}$ and $e_{4}$.

\section{References and Notes}

(1) Kramers, H. A. Physica 1940, 7, 284

(2) Chandler, D. J. Chem. Phys. 1978, 68, 2959.

(3) Montgomery, J. A., Jr.; Chandler, D.; Berne, B. J. J. Chem. Phys. 1979, 70, 4056 .

(4) Berne, B. J.; Skinner, J. L.; Wolynes, P. G. J. Chem. Phys. 1980, 73,4314 .

(5) Berne, B. J. Molecular Dynamics and Monte Carlo Simulation of Rare Events. In Multiple Time Scales; Brackbill, J. U., Cohen, B. I., Eds.; Academic Press: New York, 1985. 3172 .

(6) Straub, J. E.; Borkovec, M.; Berne, B. J. J. Chem. Phys. 1985, 83,

(7) Straub, J. E.; Borkovec, M.; Berne, B. J. J. Chem. Phys. 1986, 84, 1788.

(8) Borkovec, M.; Berne, B. J. J. Chem. Phys. 1987, 86, 2444

(9) Grote, R. F.; Hynes, J. T. J. Chem. Phys. 1980, 73, 2715.

(10) Gertner, B. J.; Bergsma, J. P.; Wilson, K. R.; Lee, S.; Hynes, J. T. J. Chem. Phys. 1987, 86, 1377.

(11) Hänggi, P. J. Stat. Phys. 1986, 42, 105.

(12) Melnikov, V. I.; Meshkov, S. V. J. Chem. Phys. 1986, 85, 1018.

(13) Pollak, E. J. Chem. Phys. 1986, 85, 865.

(14) Pollak, E.; Grabert, H.; Hänggi, P. J. Chem. Phys. 1989, 91, 4073.

(15) Nitzan, A. Adv. Chem. Phys. 1988, 70, 489.

(16) Voth, G. A.; Chandler, D.; Miller, W. H. J. Chem. Phys. 1989, 91, 7749.

(17) Ben-Nun, M.; Levine, R. D. J. Chem. Phys. 1992, 97, 8341.

(18) Ben-Nun, M.; Levine, R. D. J. Phys. Chem. 1993, 97, 2334.

(19) Ben-Nun, M.; Levine, R. D. Acc. Chem. Res. 1994, 27, 166. 251 .

(20) Hänggi, P.; Talkner, P.; Borkovec, M. Rev. Mod. Phys. 1990, 62,

(21) Zwanzig, R. Phys. Fluids 1959, 2, 12.

(22) Zwanzig, R. J. Chem. Phys. 1961, 34, 1931.

(23) Nitzan, A.; Jortner, J. Mol. Phys. 1973, 25, 713.

(24) Nitzan, A.; Silbey, R. J. Chem. Phys. 1974, 60, 4070

(25) Metiu, H.; Oxtoby, D. W.; Freed, K. F. Phys. Rev. A 1977, 15, 361.

(26) Berkowitz, M.; Gerber, R. B. Chem. Phys. 1979, 37, 369

(27) Oxtoby, D. W. Adv. Chem. Phys. 1981, 47 (part 2), 487.

(28) Oxtoby, D. W. Annu. Rev. Phys. Chem. 1981, 32, 77.

(29) Cortés, E.; West, B. J.; Lindenberg, K. J. Chem. Phys. 1985, 82, $2708-2717$.

(30) Chesnoy, J.; Gale, G. M. Adv. Chem. Phys. 1988, 70, 297.

(31) Charutz, D. M.; Berman, M.; Levine, R. D. Chem. Phys. Lett. 1989, 164, 495.

(32) Whitnell, R. M.; Wilson, K. R.; Hynes, J. T. J. Phys. Chem. 1990 , 94,8625 .

(33) Whitnell, R. M.; Wilson, K. R.; Hynes, J. T. J. Chem. Phys. 1992, 96, 5354.

(34) Dardi, P. S.; Cukier, R. I. J. Chem. Phys. 1991, 95, 98.

(35) Tuckerman, M. E.; Berne, B. J. J. Chem. Phys. 1993, 98, 7301.

(36) Bader, J. S.; Berne, B. J.; Pollak, E.; Hänggi, P. J. Chem. Phys.

1996, 104, 1111.
(37) Egorov, S. A.; Skinner, J. L. J. Chem. Phys. 1996, 105, 7047.

(38) Egorov, S. A.; Skinner, J. L. J. Chem. Phys. 1997, 106, 1034.

(39) Berne, B. J.; Pecora, R. Dynamic Light Scattering; Robert E. Krieger Publishing Co., Inc.: FL, 1990.

(40) Carmeli, B.; Nitzan, A. Phys. Rev. Lett. 1982, 49, 423.

(41) Zwanzig, R. J. Stat. Phys. 1973, 9, 215.

(42) Anderson, J. B. J. Chem. Phys. 1973, 58, 4684.

(43) Bennett, C. H. Molecular Dynamics and Transition State Theory: The Simulation of Infrequent Events. In Algorithms for Chemical Computations; Christofferson, R. E., Ed.; Am. Chem. Soc.: Washington, DC, 1977. 5188 .

(44) Straub, J. E.; Hsu, D. A.; Berne, B. J. J. Phys. Chem. 1985, 89,

(45) Straub, J. E.; Berne, B. J. J. Chem. Phys. 1985, 83, 1138.

(46) Charutz, D. M.; Levine, R. D. Chem. Phys. 1991, 152, 31.

(47) Charutz, D. M.; Levine, R. D. Chem. Phys. 1992, 159, 55.

(48) Charutz, D. M.; Levine, R. D. J. Chem. Phys. 1993, 98, 1979.

(49) Levine, R. D.; Bernstein, R. B. Molecular Reaction Dynamics and Chemical Reactivity; Oxford University Press: New York, 1987.

(50) Born, M. Mechanics of the Atom; Blackie: London, 1951.

(51) Lichtenberg, A. J.; Lieberman, M. A. Regular and Stochastic Motion; Springer-Verlag: New York, 1983.

(52) Sagdeev, R. Z.; Usikov, D. A.; Zaslavski, G. M. Nonlinear Physics: From Pendulum to Turbulence and Chaos; Harwood Academic: New York, 1989.

(53) Reichl, L. E. The Transition to Chaos; Springer-Verlag: New York, 1992.

(54) Jensen, R. V. Phys. Rev. A 1984, 30, 386.

(55) Leopold, J. G.; Richards, D. J. Phys. B 1985, 18, 3369.

(56) Casati, G.; Guarneri, I.; Shepelyansky, D. L. IEEE J. Quantum Electron 1988, QE-24, 1420.

(57) Rabani, E.; Levine, R. D.; Even, U. Ber. Bunsen-Ges. Phys. Chem. 1995, 99, 310.

(58) Rabani, E.; Levine, R. D. J. Chem. Phys. 1996, 104, 1937.

(59) Egorov, S. A.; Berne, B. J. J. Chem. Phys. 1997, 107, 6050.

(60) Child, M. S. Semiclassical Mechanics with Molecular Applications; Oxford University Press: New York, 1991.

(61) Risken, H. The Fokker-Planck Equation; Springer-Verlag: Berlin, 1989.

(62) Gardiner, C. W. Handbook of Stochastic Methods; SpringerVerlag: New York, 1994.

(63) Allen, M. P.; Tildesley, D. J. Computer Simulation of Liquids; Oxford University Press: Oxford, 1987.

(64) Tuckerman, M. E.; Martyna, G. J.; Berne, B. J. J. Chem. Phys. $1990,93,1287$

(65) Tuckerman, M. E.; Berne, B. J.; Martyna, G. J. J. Chem. Phys. 1991, 94, 6811 .

(66) Tuckerman, M. E.; Berne, B. J. J. Chem. Phys. 1991, 95, 8362.

(67) Tuckerman, M. E.; Berne, B. J.; Martyna, G. J. J. Chem. Phys. 1992, 97, 1990.

(68) Goldstein, H. Classical Mechanics; Addison-Wesley: New York, 1980.

(69) Gradshteyn, I. S.; Ryzhik, I. M. Table of Integrals, Series, and Products; Academic Press: New York, 1994.

(70) Hamming, R. W. Numerical Methods for Scientists and Engineers; McGraw-Hill: New York, 1973.

(71) Abramowitz, M.; Stegun, I. A. Handbook of Mathematical Functions; Dover: New York, 1972.

(72) Landau, L. D. Zh. Eksp. Teor. Fiz. 1937, 7, 203.

(73) Pechukas, P.; Ankerhold, J. J. Chem. Phys. 1997, 107, 2444.

(74) Agmon, N.; Kosloff, R. J. Phys. Chem. 1987, 91, 1988.

(75) Ben-Nun, M.; Levine, R. D. Chem. Phys. Lett. 1992, 192, 472. 1407.

(77) Zürcher, U.; Keyes, T. Phys. Rev. E 1997, 55, 6917.

(78) Gezelter, J. D.; Rabani, E.; Berne, B. J. J. Chem. Phys. 1997, 107, 4618.

(79) Rabani, E.; Gezelter, J. D.; Berne, B. J. J. Chem. Phys. 1997, 107 , 6867

(80) Preliminary results for a narrow set of system-bath parameters show good agreement between the mapped and Hamiltonian trajectories when higher order system-bath couplings are included. However, due to the complexity of the dynamics and the many possible coupling schemes, it is difficult to come to definite conclusions about nonlinear interactions. Therefore, a more extensive study of this system is required before any conclusion regarding the validity of the map for nonlinear couplings can be made.

(81) Rabani, E.; Berne, B. J. Unpublished.

(82) DiBartolo, B. Optical Interactions in Solids; Wiley: New York, 1968.

(83) Press, W. H.; Flannery, B. P.; Teukolsky, S. A.; Vetterling, W. T. Numerical Recipes in C; Cambridge University Press: Cambridge, 1988. 